\title{
Resultados do tratamento da Insuficiência Venosa Crônica grave com espuma de polidocanol guiada por ultrassom
}

\author{
Results of treatment of severe chronic venous insufficiency with ultrasound-guided polidocanol foam
}

Melissa Andreia de Moraes Silva', Marcelo Calil Burihan', Orlando da Costa Barros',

Felipe Nasser², José Carlos Ingrund ${ }^{3}$, Adnan Neser ${ }^{4}$

\section{Resumo}

Contexto: A insuficiência venosa crônica (IVC) em suas formas mais graves, com ulceração e lesões inflamatórias, associa-se a um tratamento cirúrgico convencional de difícil execução. A escleroterapia de veias tronculares com espuma de polidocanol é considerada uma alternativa terapêutica com resultados satisfatórios.

Objetivo: Relatar os resultados da escleroterapia com espuma de polidocanol no tratamento de pacientes com IVC de membros inferiores com úlcera ativa.

Métodos: Em um período de 48 meses, 71 pacientes (76 membros) foram submetidos à escleroterapia de veias tronculares com espuma de polidocanol a $3 \%$ guiada por ultrassom. O seguimento pós-procedimento foi feito por meio de questionário clínico, exame físico e ultrassom Doppler, realizados em sete dias, um e seis meses e, a partir de então, semestralmente. Analisou-se tempo de cicatrização da úlcera, melhora de sintomas clínicos, recanalização, recidiva dos sintomas e complicações.

Resultados: O sexo feminino correspondeu a 61\% dos casos, e a idade variou de 25 a 85 anos (média de 59 anos). O tempo médio de acompanhamento foi de 460 dias. O refluxo do sistema venoso profundo foi observado em 53,4\% dos membros. A cicatrização das úlceras ocorreu em 84,2\% dos casos, com um tempo médio de cicatrização de 37 dias. A recanalização ocorreu em 31,5\%, porém a recidiva de sintomas, com ressurgimento de úlcera, foi de 11,8\%.

Conclusão: A esclerose com espuma é efetiva e apresenta altas taxas de cicatrização de úlceras, com baixos índices de complicações, em pacientes com IVC grave.

Palavras-chave: veia safena; insuficiência venosa; úlcera varicosa; escleroterapia; ultrassonografia de intervenção.

\section{Abstract}

Background: Chronic Venous Insufficiency (CVI) in its most severe forms, with ulceration and inflammatory lesions, is associated with a conventional surgical treatment of difficult execution. Polidocanol foam sclerotherapy of saphenous veins is considered an alternative therapy with satisfactory results.

Objective: To report the results of polidocanol foam sclerotherapy in the treatment of patients with lower limb CVI with active ulcer.

Methods: Over a period of 48 months, 71 patients (76 limbs) underwent 3\% polidocanol foam ultrasound-guided sclerotherapy of saphenous vein. Follow-up was performed by means of clinical questionnaire, physical examination and Doppler ultrasound, in seven days, one and six months, and every six months thereafter. We analyzed time to ulcer healing, improvement of clinical symptoms, recanalization, recurrence of symptoms and complications.

Results: Females accounted for $61 \%$ of cases, and the age ranged from 25 to 85 years (mean 59 years). The mean follow-up was 460 days. Reflux of the deep venous system was observed in $53 \%$ of the limbs. Ulcer healing occurred in $84,2 \%$ of cases, with a mean healing time of 37 days. Recanalization occurred in $31,5 \%$, but recurrence of symptoms, with recurrence of ulcer, was $11,8 \%$.

Conclusion: Foam sclerotherapy is effective and presents high rates of ulcer healing, with low rates of complications, in patients with severe CVI.

Keywords: saphenous vein; venous insufficiency; varicose ulcer; sclerotherapy; ultrasonography, interventional.

\footnotetext{
Trabalho realizado no Hospital Santa Marcelina de São Paulo - São Paulo (SP), Brasil.

'Especialista em Cirurgia Vascular; Assistente de Cirurgia Vascular do Serviço de Cirurgia Vascular e Endovascular do Hospital Santa Marcelina - São Paulo (SP), Brasil.

2Especialista em Radiologia Intervencionista e Cirurgia Endovascular; Chefe do Departamento de Angiorradiologia e Cirurgia Endovascular do Hospital Santa Marcelina - São Paulo (SP), Brasil.

'Especialista em Cirurgia Vascular; Coordenador do Serviço de Cirurgia Vascular e Endovascular do Hospital Santa Marcelina - São Paulo (SP), Brasil.

“Especialista em Cirurgia Vascular; Chefe do Serviço de Cirurgia Vascular do Serviço Cirurgia Vascular e Endovascular do Hospital Santa Marcelina - São Paulo (SP), Brasil.

Fonte de financiamento: nenhuma.

Conflito de interesses: nada a declarar.

Submetido em: 06.11.11. Aceito em: 11.06.12.

J Vasc Bras. 2012;11(3):206-211.
} 


\section{Introdução}

Varizes dos membros inferiores são frequentes na população em geral, enquanto a estimativa de prevalência de úlcera venosa varia entre 0,2 e 1,0\% ${ }^{1}$. Esse problema interfere negativamente na qualidade de vida da população acometida e impõe gastos elevados no sistema de saúde ${ }^{2-5}$.

O tratamento convencional de pacientes portadores de formas graves de Insuficiência Venosa Crônica (IVC) pode ser de difícil execução em razão das alterações próprias da doença, como dermatoesclerose e eczema, idade avançada ou comorbidades associadas. A escleroterapia com espuma guiada por ultrassom é uma técnica simples, podendo ser realizada em ambiente ambulatorial, com uso de anestesia local, o que permite ao paciente o retorno domiciliar imediato e uma retomada precoce de atividades laborais. Pode ser repetida inúmeras vezes durante o tempo de acompanhamento do paciente nos casos de insucesso em decorrência da recanalização do vaso, tornando-se, dessa forma, uma opção promissora para o tratamento deste grupo de pacientes ${ }^{6}$.

Somente nos últimos anos, a escleroterapia com espuma guiada por ultrassom se consolidou como técnica popular na prática médica apesar de relatos de esclerose de varizes terem sido descritos desde $1950^{7}$. Com a descrição de Tessari ${ }^{8}$, uma forma mais fácil de formação da espuma foi relatada, impulsionando seu uso em todo o mundo.

O objetivo do estudo é relatar o resultado e evolução a curto e médio prazo de pacientes com úlcera venosa de membros inferiores tratados com escleroterapia com espuma de polidocanol guiada por ultrassom.

\section{Métodos}

Foram selecionados aleatoriamente 71 pacientes (76 membros) portadores de IVC com úlcera venosa ativa com mais de seis meses de evolução, já em tratamento clínico em ambulatório especializado de úlcera de membros inferiores. Todos os casos mantinham acompanhamento periódico e orientação de curativo específico (bota de Unna ou curativo simples associado à elastocompressão com uso de faixa elástica).

O período de tratamento e análise foi de junho de 2007 a maio de 2011. Todos os pacientes foram previamente orientados e assinaram o Termo de Consentimento Livre e Esclarecido para o procedimento. O trabalho foi aprovado pela Comissão Ética em Pesquisa da instituição (processo $\mathrm{n}^{\circ}$ 15/07).

Foram considerados critérios de inclusão idade entre 18 e 85 anos e presença de varizes primárias ou secundárias à Trombose Venosa Profunda (TVP) totalmente recanalizada com presença de úlcera venosa ativa. Os critérios de exclusão incluíram TVP recente ou tardia não recanalizada, gestação, trombofilia, neoplasia e insuficiência arterial periférica (índice tornozelo-braço $<0,9$ ). Foi considerado apto ao tratamento todo membro que apresentava insuficiência venosa valvular superficial, podendo apresentar associada insuficiência venosa valvular profunda ou de sistema pérfuro-comunicante. Definiu-se como refluxo o fluxo em sentido inverso com tempo superior a 0,5 segundo avaliado com o auxílio do mapeamento a cores e análise espectral, induzido por manobras de Valsalva e de compressão muscular manual distal ao posicionamento do transdutor ${ }^{9}$.

$\mathrm{O}$ tratamento escleroterápico foi realizado em regime ambulatorial. O paciente mantinha decúbito dorsal com o membro a ser tratado elevado durante a infusão da espuma. A punção foi realizada na veia safena (magna ou parva, de acordo com o mapeamento do refluxo realizado anteriormente ao tratamento) com Jelco ${ }^{\circledR}$ número $18 \mathrm{G}$ ou agulha butterfly número $21 \mathrm{G}$ ou $23 \mathrm{G}$, escolhidas de acordo com a profundidade da veia selecionada para punção, guiada com o auxílio de ultrassom, sem anestesia local na quase totalidade dos casos. Em três pacientes, foi necessário realizar anestesia local em decorrência de dor de forte intensidade na manipulação do membro. A escolha preferencial do sítio de punção foi no local onde a veia se mostrava mais próxima à pele, no terço distal de coxa ou proximal de perna. Utilizou-se uma mistura de $2 \mathrm{~mL}$ de polidocanol $3 \%$ e $8 \mathrm{~mL}$ de ar ambiente, com produção da espuma com o auxílio de torneira de três vias conectadas a duas seringas de $10 \mathrm{~mL}$ com rosca (técnica de Tessari), injetado na veia puncionada, sempre com a monitorização da ultrassonografia. Limitou-se o volume a $10 \mathrm{~mL}$ da mistura/ sessão. Realizou-se injeção isolada em veia safena na maioria dos casos (52 membros). No restante dos casos, foram realizadas duas ou mais punções (uma na veia safena em coxa e as demais no segmento distal das tributárias mais calibrosas localizadas no trajeto da veia safena magna ou parva).

Após a infusão da espuma, realizou-se a compressão excêntrica (com auxílio de chumaços de algodão ou gaze) no trajeto da veia tratada associada à compressão concêntrica do membro (por meio de faixa crepe sobreposta por meias elásticas meia coxa com cinta $20-30 \mathrm{mmHg}$ ou ataduras elásticas $15 \times 200 \mathrm{~cm}$ de baixa extensibilidade) por 48 horas. A escolha do meio de contenção foi baseada no biótipo do paciente (indivíduos obesos utilizaram a faixa elástica por indisponibilidade de tamanho adequado de meia elástica $7 / 8$ ou meia coxa com cinta) e na condição socioeconômica (alguns pacientes não puderam adquirir meias elásticas por causa do custo delas). Após esse período, o paciente foi orientado a manter apenas a contenção elástica escolhida por no mínimo 30 dias.

As variáveis iniciais avaliadas no estudo foram sexo, idade, membro acometido, quadro clínico e exame de 
ultrassonografia Doppler do membro acometido. O acompanhamento após o procedimento foi realizado por meio de questionário clínico, exame físico e ultrassom Doppler realizado em sete dias, um e seis meses e, a partir desse período, semestralmente. Todos os exames de ultrassonografia foram realizados por um dos autores, seguindo o mesmo padrão. Os pacientes foram examinados no período de acompanhamento seguindo o mesmo modelo, mantendo ortostase com o seu peso sobre o membro contralateral, com aparelho de ultrassonografia Toshiba Xario ${ }^{\circ}$ equipado com um transdutor linear. Foram estudados os segmentos venosos profundos e superficiais, interessando o fluxo, diâmetro das veias e sua compressibilidade.

Avaliou-se, durante o acompanhamento, o sucesso clínico (cicatrização da úlcera), achados ultrassonográficos (oclusão ou recanalização do vaso selecionado), recidiva dos sintomas e aparecimento de complicações. Os dados foram inseridos em protocolo de estudo previamente estabelecido. Análise estatística foi feita em planilha Microsoft Excel".

\section{Resultados}

O tempo médio de acompanhamento foi de 460 dias (mínimo 32, máximo 1.130 dias). A média de idade foi de 59 anos (25 a 85 anos), sendo $61 \%$ do sexo feminino. O membro inferior esquerdo foi acometido em $46,1 \%$ dos casos, e o

Tabela 1. Características clínicas e de tratamento dos 76 membros com úlcera venosa submetidos à escleroterapia de veias tronculares.

\begin{tabular}{lc}
\hline & $\mathrm{N}(\%)$ \\
\hline Membro acometido & \\
Direito & $41(53,9)$ \\
Esquerdo & $35(46,1)$ \\
Refluxo & \\
SVS isolada & $29(38,1)$ \\
SVS + SVP & $21(27,6)$ \\
SVS + SPC+ SVP & $20(26,3)$ \\
SVS + SPC & $6(8)$ \\
Vaso tratado & \\
VSM & $70(92)$ \\
VSP & $6(8)$ \\
Complicacões & $9(11,8)$ \\
TVP gastrocnêmias & $2(2,6)$ \\
Dor torácica & $1(1,3)$ \\
Flebite dolorosa & $6(7,8)$ \\
Recanalização com refluxo & \\
Parcial & $14(18,4)$ \\
Total & $10(13,1)$ \\
Recidiva da úlcera & $9(17,4)$ \\
\hline
\end{tabular}

SVS - sistema venoso superficial; SVP - sistema venoso profundo; SPC - sistema pérfurocomunicante; VSM - veia safena magna; VSP - veia safena parva; TVP - trombose venosa profunda. acometimento bilateral ocorreu em cinco pacientes. A maior parte dos pacientes $(53,4 \%)$ apresentava refluxo de sistema venoso profundo do membro associado à insuficiência venosa superficial, e em 26 membros observamos incompetência do sistema pérfuro-comunicante associada (Tabela 1). O sintoma mais encontrado foi dor no membro acometido, seguida de edema.

Setenta membros foram tratados com injeção da espuma em safena magna e seis membros em veia safena parva. O sucesso técnico imediato (oclusão das veias tratadas) foi de $100 \%$. O número de sessões necessárias para o sucesso técnico variou de um a quatro, e a maior parte dos tratamentos realizados foi concluída com apenas uma sessão; em dois casos, foram necessárias quatro sessões, com uma média de 1,58 sessão/membro
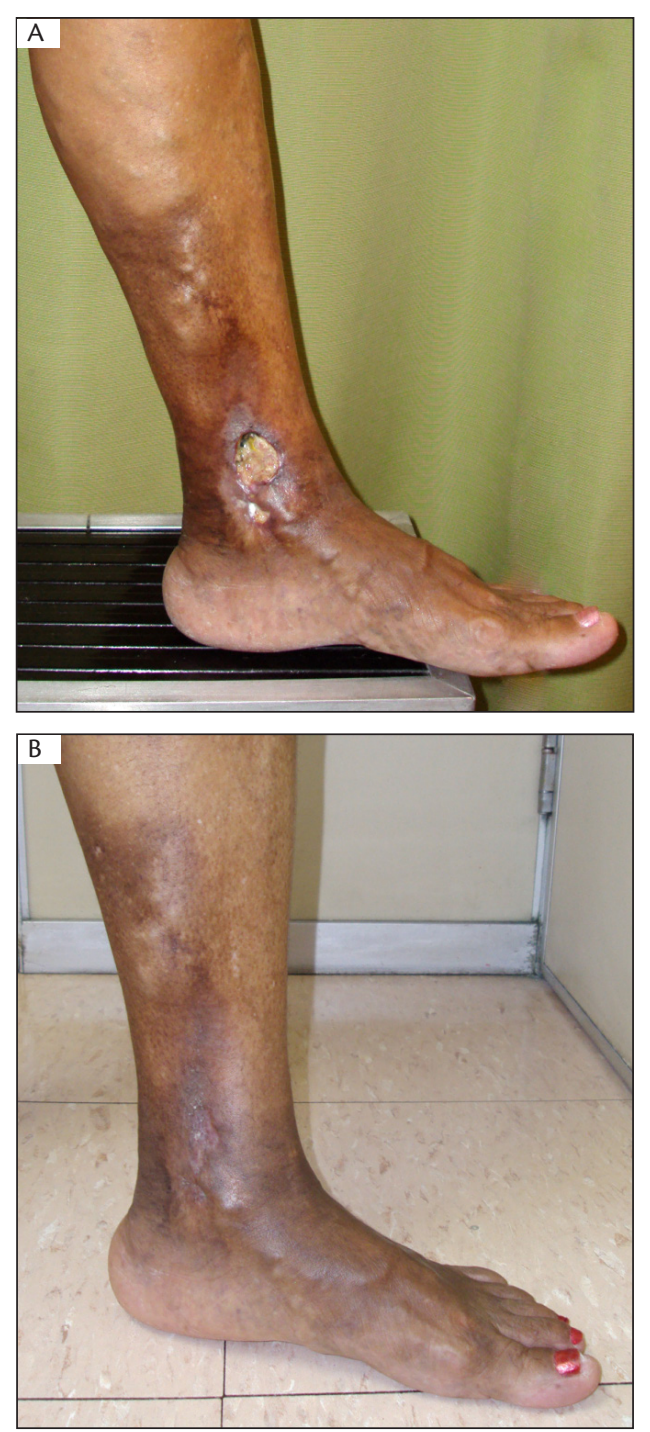

Figura 1. Paciente do sexo feminino com úlcera em face medial de perna esquerda há seis anos, com insuficiência de veia safena magna em toda sua extensão. (A) antes e (B) 30 dias após o tratamento. 
(com intervalo de sete dias entre elas). Não houve episódios de distúrbio visual ou outros sintomas neurológicos, úlcera pós-punção ou reação alérgica.

O sucesso clínico (cicatrização da úlcera) ocorreu em 64 casos (84,2\% dos membros), em um período que variou de 7 a 89 dias, com média de 37 dias (Figuras 1 e 2). Doze membros mantiveram a úlcera em atividade, com sinais de cicatrização lenta. Porém, todos os pacientes nesse grupo de falha na cicatrização relataram melhora de sintomas como dor e edema.

A maior parte dos casos tratados $(88,2 \%)$ não teve nenhum tipo de complicação. Observamos dois casos de TVP em veias gastrocnêmias $(2,6 \%)$. Um paciente referiu dor torácica momentânea após a injeção da espuma, sendo então submetido à investigação de embolia pulmonar (tomografia computadorizada de tórax). Entretanto, não houve nenhum indício radiológico de embolia, e a paciente apresentou remissão espontânea do sintoma. Seis pacientes, todos com
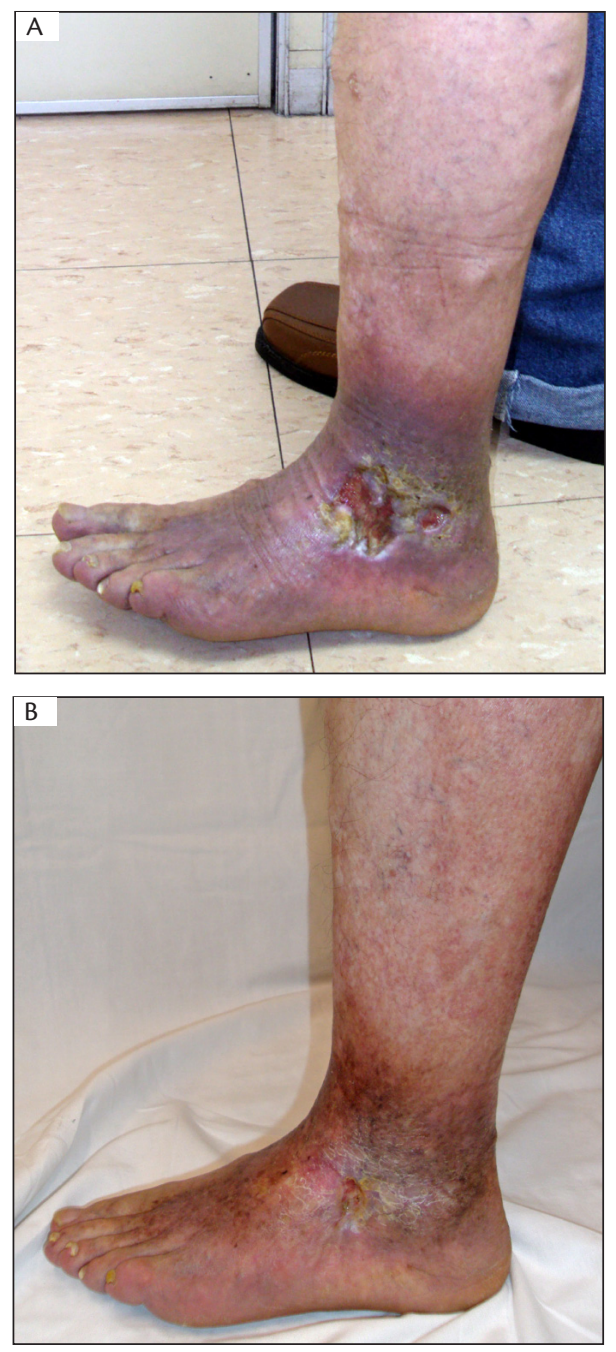

Figura 2. Paciente do sexo masculino com úlcera em face lateral de perna há dois anos. (A) antes e (B) 40 dias após duas sessões de escleroterapia. veias safenas magna com diâmetro acima de oito milímetros e varizes de grosso calibre associadas, referiram na primeira avaliação pós-procedimento sensação de dor, e notou-se presença de flebite com sinais inflamatórios exacerbados.

A recanalização parcial com refluxo foi observada em nove membros; a recanalização parcial sem refluxo, em quatro; e a recanalização total com refluxo da veia tratada (Figura 3 ), em $11^{10}$, totalizando 24 membros $(31,5 \%)$ com sinais de recanalização. Entretanto, a recidiva da úlcera somente ocorreu em nove desses 24 membros (11,8\% de todos os membros tratados). Dos nove casos de recidiva, um ocorreu em período mais tardio do acompanhamento ( $>2$ anos) e o restante, no período de 1 a 24 meses. Todos os casos de recidiva apresentavam refluxo parcial ou total da veia e foram submetidos a novo tratamento, utilizando o mesmo padrão da técnica inicial, com nova cicatrização da lesão em sete casos (Tabela 2).

\section{Discussão}

Dados da literatura sugerem que a escleroterapia com espuma guiada por ultrassom em veias tronculares superficiais em pacientes com IVC, portadores de úlcera venosa ativa, pode ser uma alternativa eficaz e atraente à cirurgia convencional nessa população6,11. A escleroterapia com espuma age ocluindo as veias superficiais incompetentes, removendo pontos de hipertensão venosa, favorecendo a cicatrização de lesões de etiologia venosa ${ }^{12}$. Cabrera descreveu uma série com

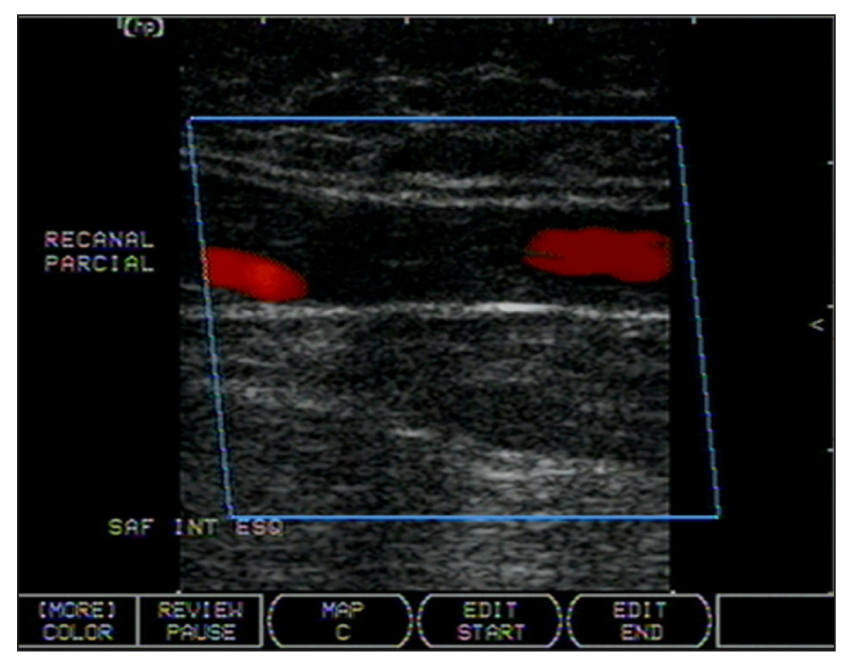

Figura 3. Recanalização parcial da veia safena magna em coxa após 12 meses do tratamento inicial.

Tabela 2. Incidência de recidivas de úlcera no período acompanhado pós-tratamento.

\begin{tabular}{lcc}
\hline Período pós-tratamento & Número de membros & Recidiva de úlcera \\
\hline$<48$ meses & 58 & 8 \\
$>48$ meses & 18 & 1 \\
\hline
\end{tabular}


116 pacientes com uma taxa de cicatrização de úlcera em seis meses de $83 \%{ }^{6}$. Bergan relatou uma série com 12 pacientes, todos submetidos a tratamento de úlceras venosas com elastocompressão sem sucesso ${ }^{11}$. Nove desses 12 pacientes tiveram as lesões cicatrizadas em período inferior a duas semanas após tratamento com esclerose com espuma.

$\mathrm{O}$ estudo ESCHAR, randomizado, com grande número de pacientes, realizado no Reino Unido, mostrou taxas gerais de cicatrização de úlcera em um período de 48 meses semelhantes entre os dois grupos estudados: elastocompressão isolada $\mathrm{x}$ cirurgia do sistema venoso superficial associada à elastocompressão $(65 \% \times 65 \%, \mathrm{p}=0,85)$, porém as taxas de recorrência de úlcera/mês foram reduzidas significativamente no grupo de compressão e cirurgia $(12 \% \times 28 \% \text {, } \mathrm{p}<0,0001)^{13}$. Em nosso grupo, obtivemos $84,2 \%$ de índice de cicatrização, valor maior que o estudo ESCHAR, mas semelhante aos outros dois estudos mencionados anteriormente. No entanto, $\mathrm{O}$ período de acompanhamento no estudo ESCHAR foi maior que no estudo atual.

A taxa de recorrência de úlcera foi de $11,8 \%$ no período estudado em nossa casuística. No estudo ESCHAR, a taxa de recorrência foi de $31 \%$ no grupo tratado com a associação compressão e cirurgia, maior que em nossa análise, porém difere de nossa casuística por apresentar acompanhamento de um número maior de pacientes, gerando significância estatística semelhante.

As complicações mais comuns encontradas são varicoflebite, seguida de trombose venosa profunda (TVP), necrose de pele e distúrbio visual. O risco de TVP decorrente do tratamento de varizes por escleroterapia guiada por ultrassom é semelhante à taxa observada em cirurgia de varizes $(0,2-1,0 \%)^{14}$.

Taxas de complicações de esclerose líquida ou espuma foram documentadas em vários estudos ${ }^{15}$. A passagem do esclerosante para o sistema venoso profundo, mesmo quando usadas pequenas doses, é comum ${ }^{16}$. Jia realizou uma metaanálise de 69 artigos com IVC tratados com escleroterapia com espuma ${ }^{17}$. Observou-se uma taxa de efeitos secundários graves entre zero e 5,7\%, com um caso de embolia pulmonar em uma série de 1.316 pacientes tratados com um percentual de trombose venosa profunda entre 0,1 e $18 \%{ }^{15,18}$. As desvantagens da técnica incluem a ocorrência de tromboembolismo venoso, que apresenta índices baixos quando comparados às técnicas convencionais. Em nossa casuística, as taxas seguem o mesmo padrão de baixa incidência.

Vários autores enfatizaram, no decorrer dos últimos anos, várias sugestões no intuito de aumentar a segurança da escleroterapia com espuma em veias tronculares ${ }^{11,19-21}$. Entre elas, incluem uso de ultrassom durante e após a injeção da espuma, posição do paciente e concentração da mistura utilizada. De acordo com o $2^{\circ}$ Consenso Europeu de Escleroterapia com Espuma, algumas medidas são recomendadas durante a realização dessa modalidade de tratamento, por exemplo, evitar a compressão imediata do trajeto aplicado após a injeção da espuma, manter sempre o controle ultrassonográfico da punção e distribuição da mistura, manter o paciente com o membro imóvel por período de três a cinco minutos (sem realizar manobra de Valsalva ou contração muscular de panturrilha) e, se houver detecção da espuma no sistema venoso profundo, realizar movimento de dorsiflexão do tornozelo ${ }^{22}$.

Vários estudos têm demonstrado a eficácia da espuma e a melhora no padrão de qualidade de vida dos pacientes submetidos ao método ${ }^{23}$. Claramente, essa é a opção mais barata no manejo de varizes de membros inferiores quando comparada com cirurgia ou técnicas de ablação térmica ${ }^{24}$.

Podemos concluir que a esclerose com espuma é efetiva e apresenta altas taxas de cicatrização de úlceras, com baixos índices de complicações no tratamento de pacientes com insuficiência venosa crônica grave.

\section{Agradecimento}

Os autores gostariam de agradecer a Alessandra Oliveira Maurício pela ajuda no atendimento e execução dos procedimentos nos pacientes envolvidos no estudo.

\section{Referências}

1. Cornwall JV, Doré CJ, Lewis JD. Leg ulcers: epidemiology and aetiology. Br J Surg. 1986;73(9):693-6. http://dx.doi.org/10.1002/bjs.1800730905

2. Kurz X, Kahn SR, Abenhaim L, et al. Chronic venous disorders of the leg: epidemiology, outcomes, diagnosis and management. Summary of an evidence-based report of the VEINES task force. Venous Insufficiency Epidemiologic and Economic Studies. Int Angiol.1999;18(2):83-102.

3. Office of Health Economics. Chronic venous disease of the leg. London, England: Office of Health Economics; 1992. p. 24-33. Report 108. PMid:8995346.

4. Ruckley CV. Socioeconomic impact of chronic venous insufficiency and leg ulcers. Angiology. 1997;48(1):67-9. PMid:8021371.

5. Phillips T, Stanton B, Provan A, Lew R. A study of the impact of leg ulcers on quality of life: financial, social, and psychologic implications. J Am Acad Dermatol. 1994;31(1):49-53.

6. Cabrera J, Redondo P, Becerra A, et al. Ultrasound-guided injection of polidocanol microfoam in the management of venous leg ulcers. Arch Dermatol. 2004;140(6):667-73. PMid:15210456.

7. Orbach EJ. Contributions to the therapy of the varicose complex. I Int Coll Surg. 1950;13(6):765-71. PMid:15428675.

8. Tessari L. Nouvelle technique d'obtention de la sclera mousse. Phlebologie.2000;53:129-33. 
9. Labropoulos N, Tiongson J, Pryor L, et al. Definition of venous reflux in lower-extremity veins. J Vasc Surg. 2003;38(4):793-8. http://dx.doi. org/10.1016/S0741-5214(03)00424-5

10. Yamaki T, Nozaki M, Iwasaka S. Comparative study of duplex-guided foam sclerotherapy and duplex-guided liquid sclerotherapy for the treatment of superficial venous insufficiency. Dermatol Surg. 2004;30(5):718-22.http://dx.doi.org/10.1111/j.1524-4725.2004.30202.x

11. Bergan J, Pascarella L, Mekenas L. Venous disorders: treatment with sclerosant foam. J Cardiovasc Surg. 2006;47(1):9-18.

12. Nicolaides A, Hussein MK, Szendro G, Christopoulos D, Vasdekis S, Clarke $\mathrm{H}$. The relation of venous ulceration with ambulatory venous pressure measurements. J Vasc Surg. 1993;17(2):414-9. http://dx.doi. org/10.1016/0741-5214(93)90426-M

13. Barwell JR, Davies CE, Deacon J, et al. Comparison of surgery and compression with compression alone in chronic venous ulceration (ESCHAR study): randomised controlled trial. Lancet 2004;363(9424):1854-9. PMid:15183623.

14. Ceulen RPM, Bullens-Goessens YIJM, Pi-Van De Venne SJA, Nelemans PJ, Veraart JC, Sommer A. Outcomes and side effects of duplex - guided sclerotherapy in the treatment of great saphenous veins with $1 \%$ versus $3 \%$ polidocanol foam: results of a randomized controlled trial with 1-year follow-up. Dermatol Surg. 2007;33(3):276-81.

15. Guex J), Allaert FA, Gillet JL, Chleir F. Immediate and midterm complications of sclerotherapy: Report of a prospective multicenter registry of 12,173 sclerotherapy sessions. Dermatol Surg. 2005;31(2):123-8. PMid:15762201.

16. Myers KA, Jolley D. Factors affecting the risk of deep venous occlusion after ultrasound-guided sclerotherapy for varicose veins. Eur J Vasc Endovasc Surg. 2008;36(5):602-5. http://dx.doi.org/10.1016/j. ejvs.2008.06.026

17. Jia X, Mowatt G, Burr JM, Cassar K, Cook J, Fraser C. Systematic review of foam sclerotherapy for varicose veins. $\mathrm{Br}$ J Surg 2007;94(8):925-36. http://dx.doi.org/10.1002/bjs.5891

18. Myers KA, Jolley D, Clough A, Kirwan J. Outcome of ultrasound - guided sclerotherapy for varicose veins: medium-term results assessed by ultrasound surveillance. Eur J Vasc Endovasc Surg. 2007;33(1):116-21. http:// dx.doi.org/10.1016/j.ejvs.2006.09.005
19. Coleridge Smith PD. Chronic venous disease treated by ultrasound guided foam sclerotherapy. Eur J Vasc Endovasc Surg. 2006;32(5):577-83. http://dx.doi.org/10.1016/j.ejvs.2006.04.033

20. Hamada T, El Hamid MA. Foam treatment for varicose veins; efficacy and safety. Sci Med J ESCME. 2006;18(1):25-40.1.

21. Kakkos SK, Bountouroglou DG, Azzam M, Kalodiki E, Daskalopoulos $M$, Geroulakos G. Effectiveness and safety of ultrasound-guided foam sclerotherapy for recurrent varicose veins: immediate results. J Endovasc Ther. 2006;13(3):357-64. http://dx.doi. org/10.1583/05-1781.1

22. Breu FX, Guggenbichler S, Wollmann JC. 2nd European Consensus Meeting on Foam Sclerotherapy 2006, Tegernsee, Germany. VASA. 2008;37 Suppl 71:1-29.

23. Figueiredo M, Araújo SP, Penha-Silva N. Ecoescleroterapia com microespuma em Varizes Tronculares Primárias. J Vasc Bras. 2006;5(3):177-83. http://dx.doi.org/10.1590/S1677-54492006000300005

24. O'Hare JL, Earnshaw JJ. Randomised clinical trial of foam sclerotherapy for patients with a venous leg ulcer. Eur J Vasc Endovasc Surg. 2010;39(4):495-9. http://dx.doi.org/10.1016/j.ejvs.2009.11.025

Correspondência Melissa Andreia de Moraes Silva Rua Conselheiro Brotero, 1065, ap. 52 CEP 01232-011 - São Paulo (SP), Brasil

E-mail: meldemoraes@hotmail.com

Contribuições dos autores Concepção e desenho do estudo: MAMS Análise e interpretação dos dados: MAMS Coleta de dados: MAMS Redação do artigo: MAMS Revisão crítica do texto: $\mathrm{AN}, \mathrm{JC}$ Aprovação final do artigo*: MAMS, MCB, OCB, JCI, AN Análise estatística: MAMS Responsabilidade geral pelo estudo: MAMS *Todos os autores leram e aprovaram a versão final submetida ao I Vasc Bras. 